Alignment of an x-Ray Imager Line of Sight in the National Ignition Facility (NIF) Target Chamber using a Diagnostic Instrument Manipulator (DIM) and Opposed Port Alignment System (OPAS)

N. Shingleton, D. Kalantar, R. Wood, T. McCarville, J. Klingmann, S. Manuel

August 6, 2012

SPIE - Optics \& Photonics

San Diego, CA, United States

August 12, 2012 through August 16, 2012 
This document was prepared as an account of work sponsored by an agency of the United States government. Neither the United States government nor Lawrence Livermore National Security, LLC, nor any of their employees makes any warranty, expressed or implied, or assumes any legal liability or responsibility for the accuracy, completeness, or usefulness of any information, apparatus, product, or process disclosed, or represents that its use would not infringe privately owned rights. Reference herein to any specific commercial product, process, or service by trade name, trademark, manufacturer, or otherwise does not necessarily constitute or imply its endorsement, recommendation, or favoring by the United States government or Lawrence Livermore National Security, LLC. The views and opinions of authors expressed herein do not necessarily state or reflect those of the United States government or Lawrence Livermore National Security, LLC, and shall not be used for advertising or product endorsement purposes. 


\title{
Alignment of an x-Ray Imager Line of Sight in the National Ignition Facility (NIF) Target Chamber using a Diagnostic Instrument Manipulator (DIM) and Opposed Port Alignment System (OPAS)
}

\author{
N. Shingleton*, D. Kalantar, R. Wood, T. McCarville, J. Klingmann, S. Manuel \\ Lawrence Livermore National Laboratory, 7000 East Ave, Livermore, CA, USA 94550
}

\begin{abstract}
The National Ignition Facility (NIF) fields multiple varieties of $\mathrm{x}$-ray imaging systems used to diagnose the implosion physics of laser-driven fusion targets. The imagers consist of time-resolved x-ray detectors coupled with a snout assembly for spatial and/or spectral imaging. The instrument is mounted onto a cart that extends into the NIF target chamber, placing it in close proximity to the target and aligning with a tight tolerance using the Opposed Port Alignment System (OPAS). The OPAS is a modified, commercial Schmidt-Cassegrain optical telescope mounted at the target chamber port, opposite the Diagnostic Instrument Manipulator (DIM). In this paper, the approach used to characterize and align the x-ray imaging instruments is described. The characterization includes offline measurements of the pinhole assembly and the detector housing. Online, deviations of the DIM, as it is inserted along rails toward the target chamber center, are characterized and related to the OPAS view. An overview of the offline measurement stations is provided along with the process to develop the relationship between the offline alignment scopes and the OPAS as a function of DIM insertion. The combination of these measurements is used to mathematically construct the predicted location of the $\mathrm{x}$-ray imager line of sight in the OPAS image space and determine the desired pinhole location to record data on a fusion experiment. The alignment accuracy of this approach will be discussed, as demonstrated with various X-ray instruments and pinhole configurations. Prepared by LLNL under Contract DE-AC52-07NA27344. LLNL-ABS-524755.
\end{abstract}

Keywords: NIF, fusion, alignment, telescope, line of sight, diagnostic, OPAS

\section{INTRODUCTION}

The National Ignition Facility (NIF) directs 192 laser beams of the world's most energetic and powerful laser to drive a $2 \mathrm{~mm}$ layered capsule of cryogenic deuterium-tritium ice to high densities and temperatures ${ }^{1}$. The extreme state of the plasma mixture provides the unique capability to study dynamic astrophysics and the main goal of demonstrating energy gain through laser driven fusion ignition. To thoroughly understand the NIF experiments, an accurate measurement of the radiation emission field and the temperature and density of target materials is required ${ }^{2}$. In order to capture these measurements, among others, a large suite of diagnostic instrumentation has been built around the 10 meter diameter vacuum chamber that houses the experiment ${ }^{3}$. A subset of the diagnostic instruments are designed to be interchangeably installed within a Diagnostic Instrument Manipulator (DIM), providing the capability to place the instrument in close proximity to the target. The majority of the instruments that ride within a DIM are streaked or time gated x-ray imaging systems coupled to pinhole snouts (Figure 1), which together provide temporal, spatial, and/or spectrally resolved properties of the target ${ }^{4}$. This paper will describe the methods to characterize the snout and detector of each instrument in order to accurately align the instrument line-of-sight (LOS) to intersect the desired object aim point.

*shingleton3@1lnl.gov; phone: 925-423-5437 


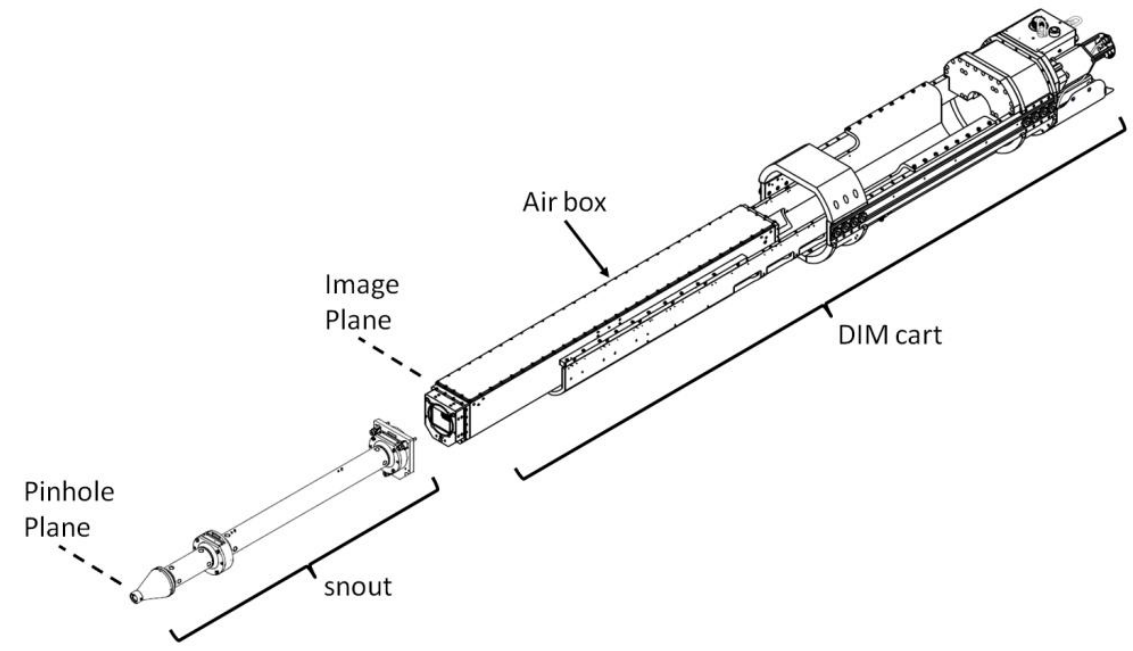

Figure 1: Schematic of a sample snout and gated x-ray detector (GXD) air box within a DIM cart. Snout offset from air box interface to show mating joint.

\section{INSTRUMENT AND ALIGNMENT SYSTEM}

The x-ray imagers that are installed onto a DIM are housed within an air box at ambient pressure and mounted onto a DIM cart (Figure 1). The DIM cart has 16 wheels that ride along rails within the DIM vessel, providing the ability to drive the cart in and out of the NIF target chamber. The DIM vessel is connected to the target chamber through a two-axis gimbal that provides pitch and yaw manipulation of the DIM by adjusting bipods that support the rear of the vessel (Figure 2). Near the front of the air box, the image plane is placed nearly $58 \mathrm{~mm}$ behind a three-vee kinematic plate that couples to a three-ball mount on the base of the snout. The snout to air box kinematic coupling is pre-loaded with three bolts to a specified torque to ensure adequate repeatability and reproducibility.

Interchangeable snouts on a DIM allow for a flexible imaging configuration to meet the needs of various experiments, each of which have a different LOS alignment tolerance. For a simple pinhole imaging snout, the pinhole tolerance, $\mathrm{E}_{\mathrm{PH}}$, can be formulated by equation 1, from the geometry, based on the image magnification, $\mathrm{M}$, the size of the detector, $\mathrm{S}_{\mathrm{det}}$, the uncertainty in the object location, $\mathrm{U}_{\text {object }}$, and the desired field of view (FOV) at the object, FOV $_{\text {object }}$.

$$
\mathrm{E}_{\mathrm{PH}}=\frac{\frac{\mathrm{S}_{\mathrm{det}}-\mathrm{M} * \mathrm{FOV}}{\text { object }_{-}}-\mathrm{M} * \mathrm{U}_{\text {object }}}{\mathrm{M}+2}
$$

The more complicated snout designs, which may include filters, mirrors, spectrometer gratings or other optical components, require a more complicated variation of equation 1 . From all of the currently designed instrument and snout configurations of a DIM, the tightest pinhole tolerances are in the range of $0.200 \mathrm{~mm}$ in each transverse dimension.

To achieve the tight alignment tolerance required to capture data during a NIF experiment, a commercial SchmidtCassegrain optical telescope has been modified to improve the optical performance and meet the functional requirements of aligning a DIM ${ }^{5}$. An Opposed Port Alignment System (OPAS) telescope is mounted on the NIF target chamber outside the port that is opposite of the DIM port. The OPAS optical platform is mounted on a manually adjustable hexapod structure (Figure 3) with differentially threaded struts that allow precision alignment of the OPAS LOS. The LOS is aligned during installation, and periodically checked, to intersect target chamber center (TCC) and the DIM rotational gimbal point. The placement and alignment of the OPAS allows the telescope to have a relatively small FOV and capture a high resolution image, while still capturing the DIM pinhole within the FOV for any pinhole-to-object standoff distance (Figure 4). 


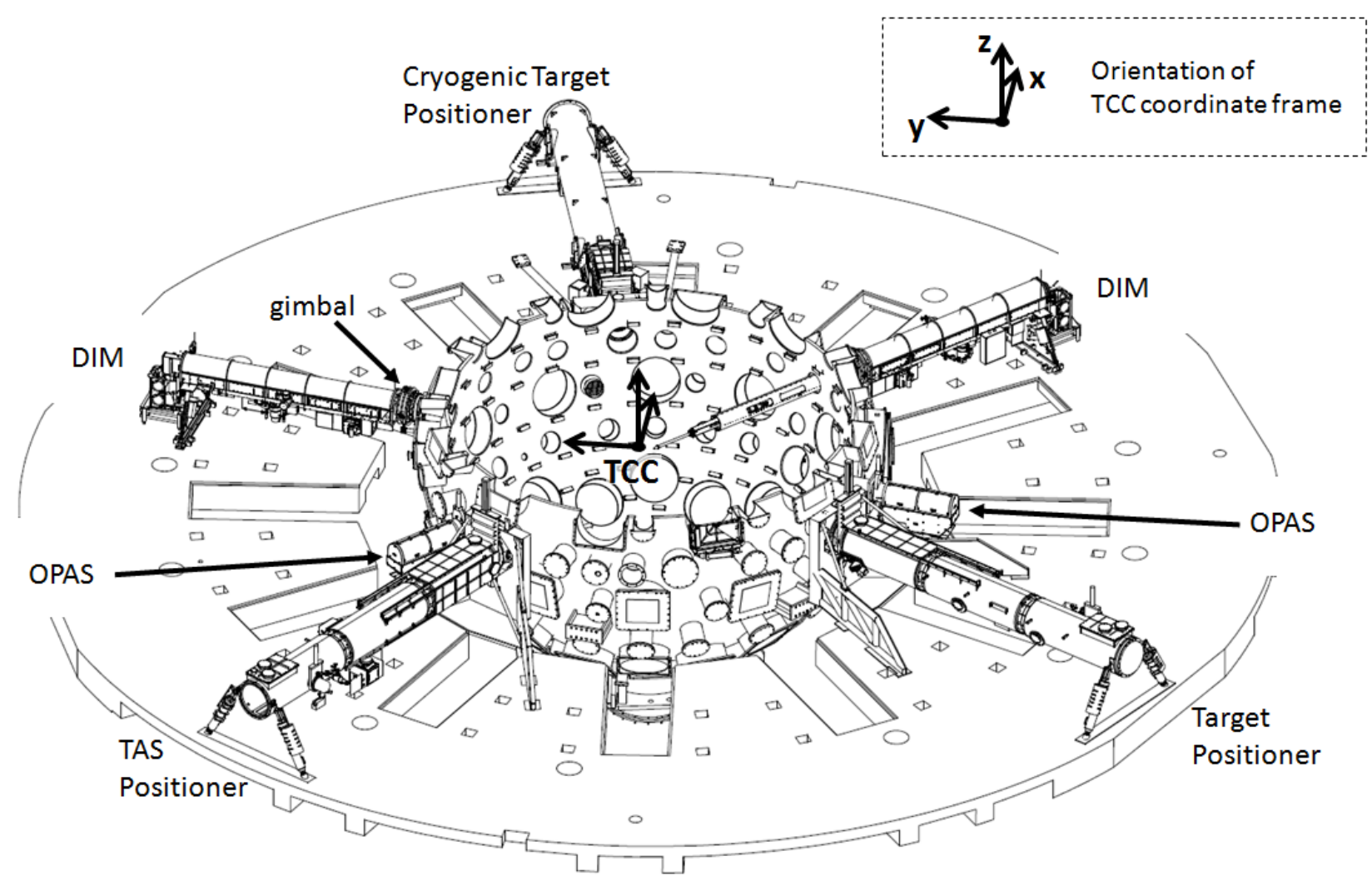

Figure 2: Cut-away NIF target chamber, showing placement of two OPAS assemblies, DIMs, TAS positioner, Target Positioners and the placement and orientation of the TCC coordinate frame.

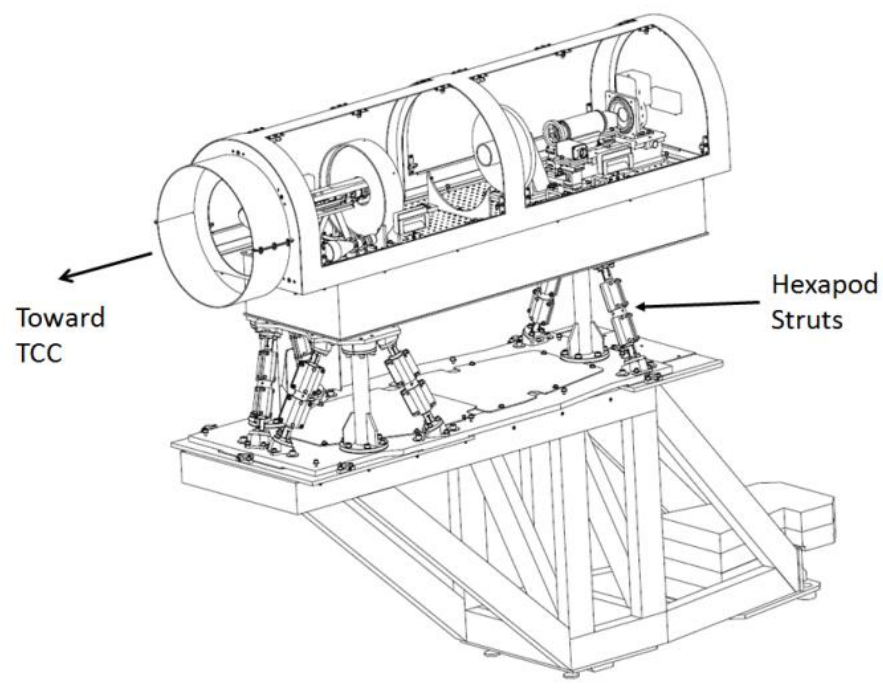

Figure 3: Schematic of the OPAS telescope supported and aligned by hexapod struts (enclosure panels removed to show telescope optical components). 

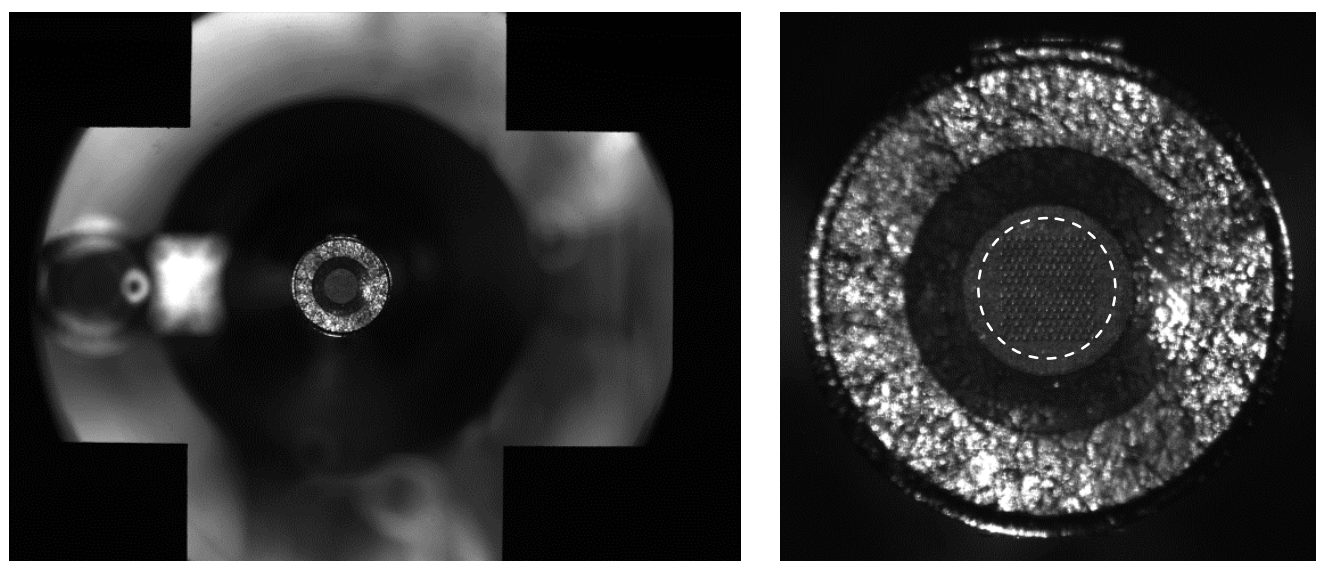

Figure 4: Full OPAS image of a snout tip (left), showing a 186-pinhole array within the NIF target chamber and the enlarged region of the image showing the pinhole array detail with the pinhole array inscribed by a dashed circle (right).

\section{NIF TARGET REGISTRATION ONTO OPAS}

From one shot to the next, a time span on the order of a day, the OPAS instability relative to the instruments used to align the NIF target, is significant, up to $0.1 \mathrm{~mm}$ at the TCC image plane. The Target Alignment Sensor (TAS) is an instrument that inserts into the target chamber on a boom and is used to align the NIF laser beams relative to the NIF target $^{6}$. The TAS is aligned relative to the TCC coordinate frame with the Chamber Center Reference System (CCRS), which is treated as the absolute reference. CCRS is a pair of specialized optical instruments dedicated to positioning the TAS in 5 degrees of freedom (DOF) ${ }^{7}$.

In order to account for the drift induced displacement of the target relative to OPAS, the new TCC recognized by the TAS must be transferred onto or registered by OPAS. To achieve target registration onto OPAS, an alignment reference fiducial is placed on the TAS hardware. Periodically, TAS is inserted into the target chamber and a small $2 \mathrm{~mm}$ spherical target is aligned to the TAS TCC coordinate frame origin. The center location of the target and the center of the TAS reference fiducial are recorded in OPAS space, which provides the spatial vector from the TAS reference fiducial to TCC (see the Appendix for a summary of the notation), as

$$
{ }^{\mathrm{OPAS}} \mathbf{v}_{\mathrm{TAS}-\mathrm{TCC}}={ }^{\mathrm{OPAS}} P_{\mathrm{TCC}}-{ }^{\mathrm{OPAS}} P_{\mathrm{TAS}} .
$$

To account for instrument drift, each time TAS is inserted and aligned for a shot, TCC can be registered onto OPAS without viewing a feature at TCC, by measuring the TAS reference fiducial point, ${ }^{O P A S} P_{\text {TAS }}$, and the pre-recorded relationship of equation 2 , by

$$
{ }^{O P A S} P_{T C C}={ }^{O P A S} P_{\text {TAS }}+{ }^{\text {OPAS }} \mathbf{v}_{\text {TAS-TCC. }}
$$

\section{INSTRUMENT CHARACTERIZATION AND LOS RECONSTRUCTION}

With TCC and the target location recorded onto OPAS, the task of aligning a DIM instrument LOS to intersect an aim point becomes an issue of understanding the diagnostic LOS. This has been accomplished by measuring the points that define the LOS, the instrument detector and snout pinhole centers, relative to a common reference coordinate frame. The common reference coordinate frame is then related to the OPAS coordinate frame in order to provide the spatial transformation required to reconstruct the diagnostic LOS in OPAS space. 
One of the reference features is composed of a plate that holds a reticule mirror. The plate has the same three ball kinematic interface as the base of a snout and the mirror is rigidly attached in an orientation approximately parallel to the kinematic interface. This feature, referred to as the mirror snout, can be thought of as having a coordinate frame rigidly attached to it, with the $\mathbf{z}$ coordinate axis orthogonal to the mirror surface and the origin coincident with the reticule (Figure 5). Before installation in a DIM, each snout is inspected on a snout alignment station, composed of a block that has the three-vee kinematic plate of a DIM air box and an auto collimating alignment telescope (Figure 6). When the mirror snout is installed on the snout alignment station block, the telescope is aligned, such that the LOS is orthogonal to the mirror snout reflective surface and intersects the reticule. The rotation of the $x-y$ plane of the mirror snout coordinate frame (MS) is defined to be parallel to the CCD pixel orientation within the telescope. With this configuration, the measurement of a point within the telescope can be easily related to the mirror snout coordinate frame by accounting for the telescope image scale. Using this method, the spatial pinhole array center point, of each snout, is measured relative to the mirror snout coordinate frame, ${ }^{M S} P_{P H}$, where the snout design length provides the $\mathbf{z}$ component.

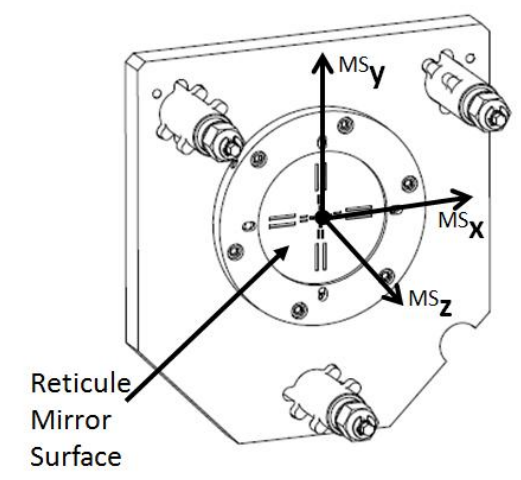

Figure 5: Mirror snout feature showing reflective reticule and reference coordinate frame.

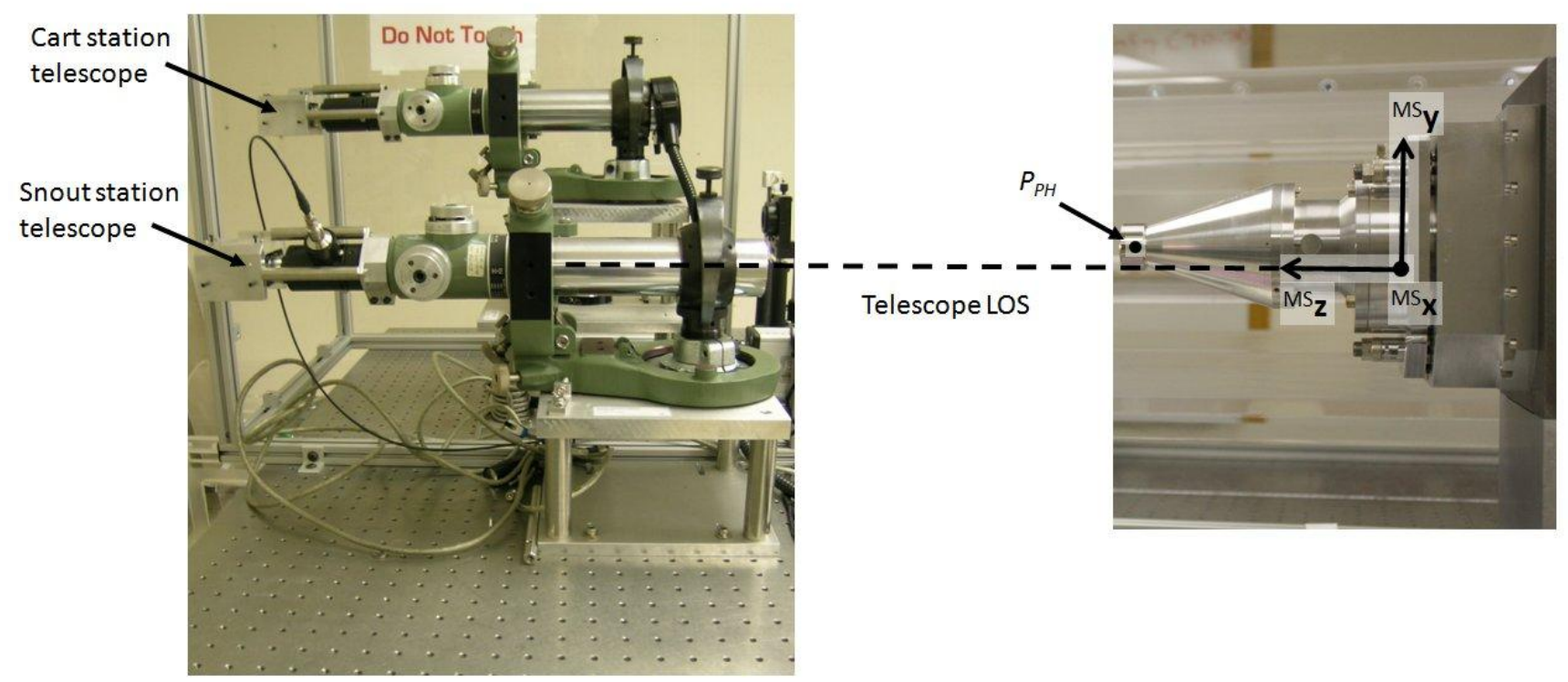

Figure 6: Snout station schematic: Snout station alignment telescope (left) and snout station kinematic block (right). Telescope LOS has been depicted by a dashed line, showing the alignment with the $\mathbf{z}$-coordinate axis of the mirror snout coordinate frame. The $\mathrm{x}$ axis is nominally coming out of the page. The mirror snout from Figure 5 can be installed in place of the 250 mm snout shown here. 
In general, it's not necessary for the telescope of the snout alignment station to be aligned to the mirror surface and reticule. In this application, the telescope is aligned to the mirror snout coordinate frame to reduce the number of measurements required for each snout. The alignment only needs to be verified each time and has shown to be sufficiently stable. Alternatively, the mirror pitch and yaw angles relative to the telescope LOS can be measured through auto collimation and the offset of the reticule can be measured to provide the spatial transformation between the snout station telescope (SS) and the mirror snout coordinate frame, ${ }^{\mathrm{SS}} \mathbf{T}_{\mathrm{MS}}$.

Once an instrument snout is mated with the kinematic mount of a diagnostic cart, the assembled rigid body LOS must be known. To complete the assembly relationship, each diagnostic cart is inspected on a cart alignment station, composed of a set of rails to hold the diagnostic cart and a second auto collimating alignment telescope. The cart station telescope is aligned to two tooling balls placed at different lengths along an alignment reference cart, nominally placing the telescope LOS to be parallel with the cart rails. The reference cart sits in the rails of the cart station with a repeatable position, to ensure the telescope alignment is consistent from one diagnostic cart measurement to the next.

A coordinate frame is defined on the cart station with the origin being coincident with the cart station telescope LOS and the $\mathrm{z}$-axis parallel to the LOS. Along the telescope LOS, the origin is defined to be at the back surface of the diagnostic cart, resulting in the distance between the cart coordinate frame $(C)$ and the wheels of the cart to be consistent, regardless of cart length. With a diagnostic cart in the cart station rails, the center of the instruments detector is measured relative to the cart coordinate frame, providing ${ }^{C} P_{d e t}$. The mirror snout from the snout alignment station is also mounted on each cart kinematic interface. The pitch and yaw angles of the mirror surface and the $\mathrm{x}, \mathrm{y}$ offset of the reticule are measured with the cart station telescope, to provide the transformation of the mirror snout coordinate frame as seen by the cart coordinate frame,

$$
{ }^{\mathrm{C}} \mathbf{T}_{\mathrm{MS}}
$$

With the measurements described thus far, the pinhole location of any snout, installed onto a cart on the cart station could be predicted by

$$
{ }^{C} P_{P H}={ }^{C} \mathbf{T}_{\mathrm{MS}}{ }^{M S} P_{P H} .
$$

However, both the pinhole and the detector location must be known, relative to OPAS, once the assembly is installed within a DIM and inserted to the correct distance from TCC. To accomplish this, the cart coordinate frame is measured relative to the OPAS coordinate frame, for each diagnostic cart, with an alignment snout that has two wire crosshairs separated by a meter. The two crosshair points on the crosshair snout are first measured on the snout station and a coordinate frame is constructed with the origin at the base crosshair and the z-axis intersecting the tip crosshair. The $x$-y axes on the crosshair snout coordinate frame are constructed to be consistent with $\mathrm{x}$-y Euler angle notation. This constructed coordinate frame provides the crosshair snout coordinate frame (xhair) as seen by the mirror snout coordinate frame,

$$
{ }^{\mathrm{MS}} \mathbf{T}_{\text {xhair }}
$$

The same crosshair snout is installed on each diagnostic cart within the NIF target chamber and the two crosshairs are measured in OPAS space with the DIM cart at insertion intervals of $100 \mathrm{~mm}$. Constructing the same coordinate frame as described above, on the crosshair snout as measured by OPAS, gives

$$
{ }^{\mathrm{OPAS}} \mathbf{T}_{\text {xhair }}\left(\mathrm{z}_{\text {insert }}\right) .
$$

This measurement captures the motion of the instrument cart in the DIM rails as the cart insertion, $z_{\text {insert }}$, is varied. Combining the spatial transformations from 4, 6 and 7, gives the transformation from the cart coordinate frame to the OPAS coordinate frame as a function of insertion, as

$$
{ }^{\mathrm{OPAS}} \mathbf{T}_{\mathrm{C}}\left(\mathrm{z}_{\text {insert }}\right)={ }^{\mathrm{OPAS}} \mathbf{T}_{\text {xhair }}\left(\mathrm{z}_{\text {insert }}\right){ }^{\text {xhair }} \mathbf{T}_{\mathrm{MS}}{ }^{\mathrm{MS}} \mathbf{T}_{\mathrm{C}}
$$


Using equation 8, the diagnostic LOS can be mathematically constructed in OPAS space by transforming the pinhole and detector point with equation 9 and 10, respectively.

$$
\begin{gathered}
{ }^{O P A S} P_{P H}={ }^{\mathrm{OPAS}} \mathbf{T}_{\mathrm{C}}\left(\mathrm{z}_{\text {insert }}\right){ }^{\mathrm{C}} \mathbf{T}_{\mathrm{MS}}{ }^{M S} P_{P H} \\
{ }^{O P A S} P_{\text {det }}={ }^{\mathrm{OPAS}} \mathbf{T}_{\mathrm{C}}\left(\mathrm{z}_{\text {insert }}\right){ }^{C} P_{\text {det }}
\end{gathered}
$$

The LOS from equations 9 and 10 is arbitrarily located at the location it would be placed if the pitch and yaw of the DIM gimbal were set at the same angles as during the crosshair snout measurement with OPAS. To calculate the desired location of the LOS to achieve alignment, the arbitrarily located LOS is projected to the TCC object plane, giving ${ }^{O P A S} P_{\text {aim,actual }}$. The DIM gimbal yaw $\left(\psi_{\text {yaw }}\right)$ and pitch $\left(\gamma_{\text {pitch }}\right)$ correction angles are calculated, assuming small angles, by equation 10 , where $\mathrm{d}_{\text {gimbal-TCC }}$ is the nominal distance from the DIM gimbal to TCC.

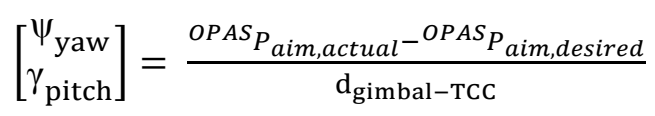

With the required yaw and pitch correction angles of the DIM gimbal, the LOS can be transformed to the aligned spatial location. First, the LOS points are transformed to a coordinate frame oriented parallel to the OPAS coordinate frame, but with the origin coincident with the DIM gimbal, ${ }^{\mathrm{OPAS}} \mathbf{T}_{\mathrm{DIM}}$, where the transformation is defined by the nominal relationship. Since the OPAS telescope was originally aligned to have the LOS intersect the DIM gimbal, the uncertainty induced into the calculation due to a deviation from nominal is small. The LOS points are then rotated about the gimbal by the correction angles from equation 11, before being transformed back into OPAS space. The result, shown in equation 12 , gives the desired location of the pinhole to achieve proper alignment.

$$
{ }^{O P A S} P_{P H}={ }^{\mathrm{OPAS}} \mathbf{T}_{\mathrm{DIM}} \mathbf{R}_{\mathrm{X}}\left(\gamma_{\text {pitch }}\right) \mathbf{R}_{\mathrm{Y}}\left(\psi_{\text {yaw }}\right){ }^{\mathrm{DIM}} \mathbf{T}_{\mathrm{OPAS}}{ }^{O P A S} P_{P H}
$$

For each NIF shot, this process is used to pre-calculate the aligned location of the pinhole on OPAS, ${ }^{O P A S} P_{P H}$, and the pinhole is placed accordingly, by adjusting the DIM about the gimbal. Although the detector point on OPAS, ${ }^{O P A S} P_{d e t}$, can be calculated, the detector is not visible in the OPAS view and is not used or needed for the in-situ instrument alignment.

\section{RESULTS}

The performance of the characterization and alignment method described in this paper has been evaluated by analyzing post shot diagnostic images. Usually the image center is found by using a non-uniform pinhole array pattern in the snout, which can be easily identified on the image (Figure 6). Neglecting roll, there are 5 DOF that must be known to achieve alignment of the diagnostic LOS, the insertion and the two translational components of both the pinhole and the detector. The DIM only has 3 DOF of manipulation, insertion and pitch and yaw about the gimbal, the other 2 DOF are fixed based on the hardware assembly. Since the diagnostic image only provides 2 DOF of feedback regarding the resulting alignment, in order to quantify the results it's assumed that the full alignment error is due to misplacing the LOS with the DIM manipulation about the gimbal. This implies that the misalignment is due to a parallel, but offset actual LOS compared to the theoretical, perfectly aligned LOS. Table 1 summarizes the alignment results, categorized by the instrument magnification and the overall results. The overall root-mean square (RMS) error in both the $\mathrm{x}$ and $\mathrm{y}$ direction equates to $0.170 \mathrm{~mm}$. Even though only 27 data points have been collected, the equivalent standard errors in the 2 dimensions suggests that enough statistics have been collected to sufficiently estimate the performance of the method. 

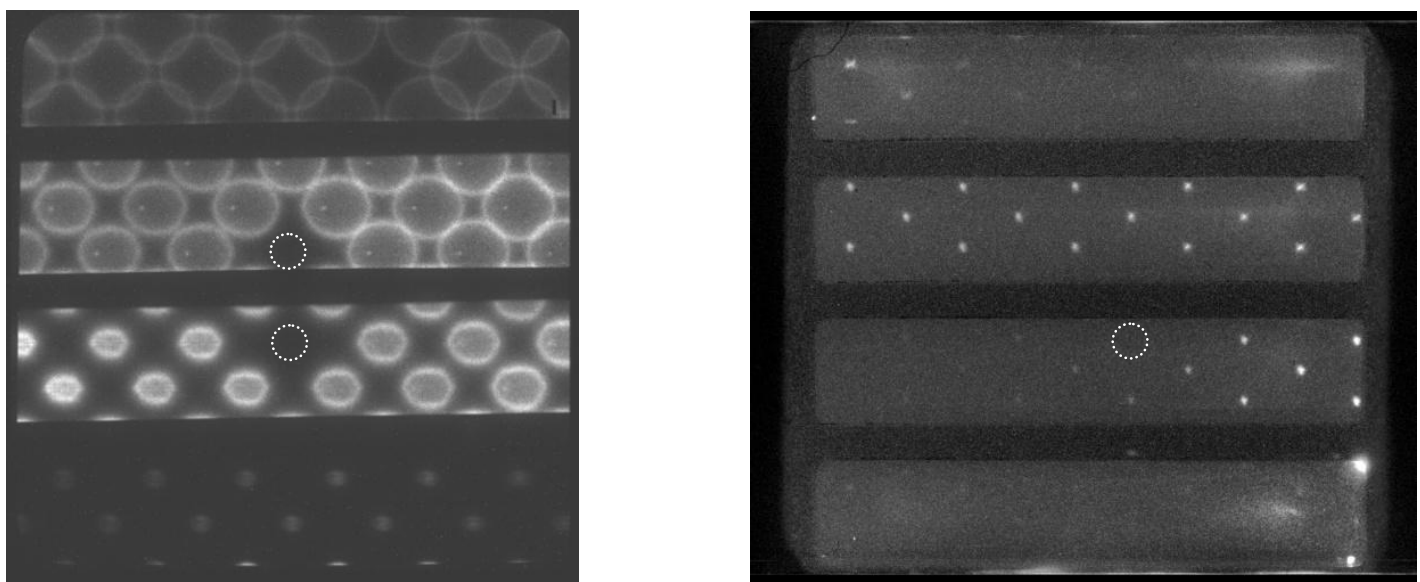

Figure 6: X-ray diagnostic images of an imploding NIF target captured with a gated x-ray detector (GXD) (left) and a hardened gated $\mathrm{X}$-ray imager (hGXI) (right). Both images show the non-uniform pattern of pinhole arrays used to evaluate alignment performance.

The missing pinhole images are indicated by the dotted circles.

Table 1: Alignment statistics from application of the described method. Results categorized by instrument magnification.

\begin{tabular}{|c|c|c|c|c|c|c|}
\hline \multirow{2}{*}{ Magnification } & \multicolumn{3}{|c|}{ RMS Misalignment (mm) } & \multicolumn{2}{c|}{$\begin{array}{c}\text { Maximum } \\
\text { Misalignment (mm) }\end{array}$} & $\begin{array}{c}\text { \# of } \\
\text { measurement } \\
\text { sets }\end{array}$ \\
\cline { 2 - 6 } & $\mathbf{X}$ & $\mathbf{Y}$ & Magnitude & $\mathbf{X}$ & $\mathbf{Y}$ & 2 \\
\hline $4 \mathrm{X}$ & 0.080 & 0.110 & 0.140 & 0.110 & 0.130 & 6 \\
\hline $9 \mathrm{X}$ & 0.070 & 0.130 & 0.150 & 0.110 & 0.200 & 11 \\
\hline $12 \mathrm{X}$ & 0.250 & 0.230 & 0.340 & 0.590 & 0.580 & 8 \\
\hline $15 \mathrm{X}$ & 0.060 & 0.100 & 0.120 & 0.100 & 0.180 & 27 \\
\hline Overall & 0.170 & 0.170 & 0.240 & 0.590 & 0.580 & \\
\hline
\end{tabular}

The 12X magnification snout configuration has higher misalignment results as compared to the other configurations. Of the 22 measurements, 11 in each of the 2 dimensions, for the 12x magnification, there are 3 values that are significantly larger than the rest, with a maximum of $0.590 \mathrm{~mm}$. This indicates the values may be statistical outliers caused by an off normal event, such as operator error. By eliminating the higher numbers, the remaining misalignments are less than $0.250 \mathrm{~mm}$ in each dimension, with an RMS of $0.130 \mathrm{~mm}$ and $0.150 \mathrm{~mm}$ in $\mathrm{x}$ and $\mathrm{y}$, respectively. The pinhole arrays used with the $12 \mathrm{x}$ and $15 \mathrm{x}$ magnification snouts are designed to spatially oversample and loosen the tolerance to be greater than $0.7 \mathrm{~mm}$, so that even the higher misalignments did not miss data.

The misalignments in the $\mathrm{x}$ direction appear to nearly fall within a Gaussian distribution, with an average of around $-0.05 \mathrm{~mm}$. The non-zero average Gaussian distribution, suggests that there may be a bias of $-0.05 \mathrm{~mm}$ introduced somewhere within the process. A possible step that could be contributing this bias is an error in registering TCC onto OPAS. If the reason for the bias can be located and removed, the alignment results may improve. The misalignments in the $\mathrm{y}$ direction are regularly larger than in the $\mathrm{x}$ direction, this may be due to a decrease in the repeatability of the hardware in the y direction, as a result of gravity forces.

\section{CONCLUSIONS}

A unique method has been developed to routinely characterize and align the LOS of pinhole instruments for a unique application of recording $\mathrm{x}$-ray data from fusion experiments. These methods could be directly transferred to similar applications requiring characterization of kinematic hardware assemblies, when the assembly must be characterized in pieces with a series of simple steps and accurately known after assembly. The resulting performance, for this application, has shown to provide sub millimeter alignment accuracy, with the majority of instruments aligned to better than or equal to $0.250 \mathrm{~mm}$ in each dimension. The biased alignment error in the horizontal direction will continue to be 
monitored and verified by analyzing future alignment performance. In the near future, the accuracy of registering TCC onto OPAS will be improved by increasing the quality and contrast of the alignment fiducials and the image processing routines to measure their location. This may eliminate the horizontal bias, if the TCC registration process is the cause. With the existing process as it is, DIM snouts are regularly aligned within tolerance, successfully providing the data to advance the understanding of laser driven inertial confinement fusion at the NIF.

\section{APPENDIX}

\section{Notation summary}

In multiple cases throughout this method, points are measured with the use of a telescope imaging system and equated to 3D spatial measurements by accounting for the pixel scale of the image and using the design length to capture the third component. Unless stated otherwise, the notation in this paper is referring to 3-dimensional (3D) spatial information. Therefore, points are spatial points, which are denoted by uppercase italic variables, where the subscript indicates the unique instance of a point, for example point $\mathrm{i}, P_{i}$, is different than point $\mathrm{j}, P_{j}$. A point exists in space, but in order to assign unique coordinates to the point, it must also be referenced to a specific coordinate frame. The coordinate frame that a point is referenced relative to is denoted in the superscript, such as ${ }^{W} P_{i}$, the coordinate of point $\mathrm{i}$ as seen by the W coordinate frame. Lowercase bold variables represent a vector, where ${ }^{\mathrm{W}} \mathbf{v}_{\mathrm{j}-\mathrm{i}}={ }^{\mathrm{w}} P_{j}-{ }^{\mathrm{W}} P_{i}$, is the displacement from point $\mathrm{i}$ to point $\mathrm{j}$ relative to coordinate frame, $\mathrm{W}$.

A spatial point or vector can be transformed between coordinate frame spaces by a homogeneous coordinate transformation, denoted by a bold uppercase $\mathbf{T}$. That is, ${ }^{\mathrm{W}} \mathbf{T}_{\mathrm{N}}$, is the homogeneous coordinate transformation from $\mathrm{N}$ to $\mathrm{W}$, which can otherwise be thought of as the spatial location and orientation of coordinate frame $\mathrm{N}$ as seen by W. The transformation matrix, ${ }^{\mathrm{W}} \mathbf{T}_{\mathrm{N}}$, is composed of the rotation matrix, ${ }^{\mathrm{W}} \mathbf{R}_{\mathrm{N}}$, and translation vector, ${ }^{\mathrm{W}} \mathbf{d}_{\mathrm{N}-\mathrm{w}}$, and can be expanded as

$$
{ }^{\mathrm{W}} \mathbf{T}_{\mathrm{N}}=\left[\begin{array}{cc}
{ }^{\mathrm{w}} \mathbf{R}_{\mathrm{N}} & { }^{\mathrm{w}} \mathbf{d}_{\mathrm{N}-\mathrm{W}} \\
\mathbf{0}^{T} & 1
\end{array}\right]=\left[\begin{array}{cccc}
\mathrm{w}_{\mathbf{x}_{\mathrm{N}}} & { }^{\mathrm{w}}{ }_{\mathbf{y}_{\mathrm{N}}} & { }^{\mathrm{w}} \mathbf{z}_{\mathrm{N}} & { }^{\mathrm{w}} \mathbf{d}_{\mathrm{N}-\mathrm{w}} \\
0 & 0 & 0 & 1
\end{array}\right]
$$

where ${ }^{\mathrm{W}} \mathbf{x}_{\mathrm{N}},{ }^{\mathrm{w}} \mathbf{y}_{\mathrm{N}}$, and ${ }^{\mathrm{w}} \mathbf{z}_{\mathrm{N}}$ are the coordinate axis vectors of coordinate frame $\mathrm{N}$ as seen by $\mathrm{W}$. The inverse of a transformation matrix provides the inverse spatial relationship. Therefore, a spatial point $i$ in the $\mathrm{W}$ coordinate frame, ${ }^{W} P_{i}$, can be transformed to the $\mathrm{N}$ coordinate frame by

$$
{ }^{N} P_{i}={ }^{\mathrm{N}} \mathbf{T}_{\mathrm{W}}{ }^{W} P_{i}={ }^{\mathrm{W}} \mathbf{T}_{\mathrm{N}}{ }^{-1}{ }^{W} P_{i}
$$

Lastly, the following rotation matrices of equations A.3.1 through A.3.3 denote a rotation about the current $\mathbf{x}, \mathbf{y}$, and $\mathbf{z}$ coordinate axis, respectively.

$$
\begin{aligned}
\boldsymbol{R}_{x}(\theta) & =\left[\begin{array}{ccc}
1 & 0 & 0 \\
0 & \cos \theta & -\sin \theta \\
0 & \sin \theta & \cos \theta
\end{array}\right] \\
\boldsymbol{R}_{y}(\gamma) & =\left[\begin{array}{ccc}
\cos \gamma & 0 & \sin \gamma \\
0 & 1 & 0 \\
-\sin \gamma & 0 & \cos \gamma
\end{array}\right] \\
\boldsymbol{R}_{z}(\xi) & =\left[\begin{array}{ccc}
\cos \xi & -\sin \xi & 0 \\
\sin \xi & \cos \xi & 0 \\
0 & 0 & 1
\end{array}\right]
\end{aligned}
$$




\section{REFERENCES}

[1] Moses, E., et Al., "The National Ignition Facility: Status and Plans for Laser Fusion and High-Energy-Density Experimental Studies," Fusion Science and Technology, V. 43, p. 420 (May 2003).

[2] Back, C. A., Bauer, J. D., Hammer, J. H., Lasinski, B. F., Turner, R. E., Rambo, P. W., Landen, O. L., Suter, L. J., Rosen, M. D., and Hsing, W. W., "Diffusive, supersonic x-ray transport in radiatively heated foam cylinders," Physics of Plasmas 7, no. 5, 2126 (May 2000).

[3] Dewald, E. L., Glenzer, S. H., Landen, O. L., et Al., "First laser-plasma interaction and hohlraum experiments on the national ignition facility," Plasma Phys Control Fusion, 47, p. B405 (2005).

[4] Pak, A., Divol, L., Weber, S., Doppner, T., Kyrala, G. A., Kilne, J., Izumi, N., et al., "Diagnosing radiative shocks from deuterium and tritium implosions on NIF," Review of Scientific Instruments, 83, p. 10E507 10E507-3 (Oct. 2012).

[5] Manuel, A. M., McCarville, T., Seppala, L., Klingmann, J., Kalantar, D., "Opposed Port Alignment System (OPAS): A commercial astronomical telescope modified for viewing the interior of the NIF target chamber," Proc. SPIE 8491 (2012).

[6] Di Nicola, P., Kalantar, D. H., McCarville, T. J., et Al., "Beam and target alignment at the National Ignition Facility using the Target Alignment Sensor (TAS)," Proc. SPIE 8505 (2012).

[7] Kalantar, D., et Al., "An overview of target and diagnostic alignment at NIF," Proc. SPIE 8505 (2012). 